\title{
Effects of Release Modifier and Annealing on Polyvinyl Acetate-Polyvinyl Pyrrolidone Microspheres Containing Diclofenac Sodium
}

\author{
Md. Saiful Islam and Reza-ul J alil \\ Department of Pharmaceutical Technology, Faculty of Pharmacy, University of Dhaka, \\ Dhaka-1000, Bangladesh
}

\begin{abstract}
This study was conducted to microencapsulate water soluble diclofenac sodium (DS), a potent NSAID advocated for use in painful, inflammatory, and certain non-rheumatic conditions. To modify its release pattern, polyvinyl acetate-polyvinyl pyrrolidone based matrix polymer, Kollidon ${ }^{\circledR}$ SR (KSR) was used as wall material. Microspheres were prepared by water-in-oil (W/O) emulsion solvent evaporation technique using KSR while maintaining core to polymer ratio at 1:1. Two release modifiers namely polyethyleneglycol 6000 (PEG 6000) and talc at four different concentrations (3\%, 5\%, $10 \%$ and $15 \% \mathrm{wt} / \mathrm{wt}$ of the wall material) were used to investigate their effect on microsphere properties and release kinetics of the drug. Optimized batches were characterized according to particle size distribution, surface morphology, differential scanning thermogram, and in vitro release of the drug. In vitro dissolution study was conducted in phosphate buffer solution of $\mathrm{pH} 7.2$ for 8 hours. Microsphere sizes were within the range of $497-922 \mu \mathrm{m}$. No interaction between drug and excipients was observed. Characteristic changes in the microsphere surface were observed due to the presence of PEG 6000 and talc. All optimized batches showed good controlled release property with immediate burst release. Annealing (heating the microspheres at $60^{\circ} \mathrm{C}$ for 24 hours) was found effective in controlling the immediate burst release of the drug. Case I or fickian-type release mechanism was found predominant in case of all formulated microspheres.
\end{abstract}

Key words: Diclofenac Sodium, Microsphere, Kollidon ${ }^{\circledR}$ SR, Solvent Evaporation Technique, Talc, Polyethyleneglycol 6000, Annealing.

\section{INTRODUCTION}

In case of oral route, non-steroidal antiinflammatory drugs (NSAIDs) are usually good candidates for the development of controlled release drug delivery systems. It has been observed previously that treatment with NSAIDs always associates with adverse effects in the gastric mucosa. ${ }^{1}$ Especially the drugs with short half-life require frequent daily administration, which eventually causes more gastric-disturbance complains. ${ }^{2}$ To reduce or alleviate the irritation of gastrointestinal mucosa, multiparticulate systems particularly microspheres have been proved successful for the preparation of oral controlled release formulations. ${ }^{3-5}$

Correspondence to: Md. Saiful Islam

Tel: (088)-01732-073108

E-mail: msipharmacy@du.ac.bd

Dhaka Univ. J. Pharm. Sci. 12(1): 41-50, 2013 (June)
Diclofenac sodium (DS) is one of the most potent NSAID used for symptomatic treatment of rheumatoid arthritis, osteoarthritis and ankylosing spondilitis. Gastrointestinal side effects such as bleeding, ulceration or perforation of intestinal wall are commonly seen. ${ }^{6}$ Its biological half-life has been reported as 1-2 $\mathrm{h}$ following oral dosing. ${ }^{7}$ Due to a short biological half-life and associated adverse effects, it is considered as an ideal candidate for controlled drug delivery. ${ }^{3,5,7}$

In this study, DS microspheres were prepared using the emulsion solvent evaporation method, which is a simultaneous process that combines spherical agglomeration and microencapsulation of the drug. The technique was selected due to its simplicity, low cost, success with many drugs and the 
production of microspheres of relatively high drug loading. ${ }^{8-10}$

Polyvinyl acetate and polyvinyl pyrrolidone based matrix polymer (Kolidon ${ }^{\circledR}$ SR) (KSR) was used as the wall material for microsphere fabrication. KSR is a water insoluble polymer and has been used in preparation of sustained release tablets, pellets, and granules. ${ }^{11-14}$ But in this study, KSR was used as the wall material to prepare sustained release microspheres of DS as it has been shown that KSR can also be a good polymer to be used for such purpose. ${ }^{15,16}$

Annealing is a good technique to control the release of drugs from different types of dosage forms. It is a technique where dosage forms are treated at different temperatures both below and above the glass transition temperatures ( $\mathrm{Tg}$ ). If the annealing temperature is above the $\mathrm{Tg}$, there is a possibility of more flexibility or elasticity between the polymer molecules and eventually the polymer molecules become fused. As a result, drug release is affected due to this fusion of the polymer molecules. Annealing technique has been proved successful in controlling drug release from different types of dosage forms prepared with different polymers. ${ }^{10,17-19}$ Therefore, the aim of this study was (i) to observe the alteration in the release kinetics of DS from KSR microspheres due to presence of PEG 6000 and talc, and (ii) to investigate the effect of annealing on release kinetics of the drug.

\section{MATERIALS AND METHODS}

Diclofenac sodium (DS) was received as a gift sample from Square Pharmaceuticals, Bangladesh. Voltalin SR $100 \mathrm{mg}$ tablets were kindly provided by NOVARTIS, Bangladesh. Kollidon ${ }^{\circledR}$ SR (BASF, Germany), talc (Whittaker, Clark and Daniels Inc, USA), polyethyleneglycol (PEG 6000) (BASF, Germany), span 60 (BDH Chemicals Ltd., England), methanol (Merck, Germany), light liquid paraffin (LLP) (Merck, Germany), petroleum ether of 40-60 ratios (Merck, Germany) were also used as received.

Preparation of DS microspheres. Microspheres were prepared using W/O emulsion solvent evaporation technique, which was slightly modified form of the technique used by Jalil and Nixon. ${ }^{10}$

Briefly, $100 \mathrm{gm}$ of light liquid paraffin (LLP) containing $1 \%$ (wt/wt of the continuum) of span 60 (external phase) was taken in a beaker. 4 gm of KSR and $4 \mathrm{gm}$ of DS were dissolved in $20 \mathrm{gm}$ of methanol (internal phase) with the help of a vortex mixer (Digisystem laboratory instruments inc. Taiwan). Internal phase was then dispersed within the external phase with the help of a high speed stirrer (Heidolph No. 5011, Heidolph, Germany) at $3000 \mathrm{rpm}$ at the ambient conditions. After 3 hours of stirring, hard and spherical microspheres were obtained. Microspheres were then recovered by filtration followed by washing with petroleum ether (40:60) for three times. Microspheres were finally sieved (Endecott's Test Sieve, Endecotts Limited, England) to remove any lump present and stored in a desiccator until further use. This batch was considered as "blank." PEG 6000 and talc were also incorporated in the internal phase according to table 1.

Particle size distribution study. Microsphere size was determined in a particle size analyzer, Mastersizer 2000 (Malvern, UK) using Dry Dispersion technique. Average particle size was expressed as volume mean diameter (D [4, 3]), population mean diameter $(\mathrm{D}[3,2])$ and span. Span is width of distribution based on the $10 \%, 50 \%$ and $90 \%$ quantile and is determined by-

$$
\operatorname{Span}=\frac{\mathrm{D}[\mathrm{v}, 0.9]-\mathrm{D}[\mathrm{v}, 0.1]}{\mathrm{D}[\mathrm{v}, 0.5]}
$$

where, $\mathrm{d}(0.1)$ indicates $10 \%$ volume distribution is below $\mathrm{X} \mu \mathrm{m}, \mathrm{d}(0.5)$ indicates $50 \%$ volume distribution is above $\mathrm{Y} \mu \mathrm{m}$ and $\mathrm{d}(0.9)$ indicates $90 \%$ volume distribution is below $\mathrm{Z} \mu \mathrm{m}$.

Surface morphology study. Microsphere surface morphology was examined in a scanning electron microscope (SEM) (S-3400N, Hitachi, Japan). SEM images at two magnifications (X200 and X500) were taken.

Differential scanning calorimetric study. Thermal behavior of the compounds was examined in a differential scanning calorimeter (DSC), DSC Q100 
(TA instruments, New Castle, USA) where 5-6 mg sample was placed in an aluminum pan at a heating rate of $10^{\circ} \mathrm{C} / \mathrm{min}$ with purging of dry nitrogen at a constant rate of $50 \mathrm{ml} / \mathrm{min}$. Indium/Zinc standards were used to calibrate the DSC temperature and enthalpy scale.

Drug loading and encapsulation efficiency determination. A Shimadzu UV-VIS Spectrophotometer (UV mini-1240, Shimadzu Corp., Kyoto, Japan) set at $276 \mathrm{~nm}$ was used to measure the absorbance of aqueous solution of DS (0 to $20 \mu \mathrm{g} / \mathrm{ml}$ ) prepared by using $0.2 \mathrm{M}$ molar phosphate buffer solution ( $\mathrm{pH}$ 7.2). A linear line was obtained while absorbance values were plotted against concentrations $\left(\mathrm{R}^{2}>0.996\right)$.

Drug loaded microspheres from each batch were finely powdered in a glass mortar and $10 \mathrm{mg}$ powder was taken in a volumetric flask. A clear solution was made using the phosphate buffer of $\mathrm{pH} 7.2$ with the help of a sonicator (Power Sonic 505, Hwashin Technology co., Seoul, Korea). Then the solution was filtered through $0.45 \mu \mathrm{m}$ filter (Microsart ${ }^{\circledR}$ Hannover, Germany) and was analyzed spectrophotometrically for drug content. Drug loading was calculated using the total weight of DS present in a particular batch. ${ }^{8,20}$

To determine the encapsulation efficiency of the prepared microspheres, weight of DS theoretically contained in the microspheres was compared with the weight actually obtained from the drug content studies, i.e., the quantity loaded into the microspheres formulated. ${ }^{21}$

\%Drug loading $=\frac{\mathrm{W}_{\mathrm{d}}}{\mathrm{W}_{\mathrm{m}}} \times 100$
\%Encapsulation $=\frac{\mathrm{D}_{\mathrm{p}}}{\mathrm{D}_{\mathrm{t}}} \times 100$

where, $\mathrm{W}_{\mathrm{d}}$ is the weight of DS present in $\mathrm{W}_{\mathrm{m}}$ gm of microspheres and $D_{p} \& D_{t}$ were the practical and theoretical drug content in the microspheres, respectively.
Annealing of the microspheres. Microspheres were taken in screw-cap vials. The vials were kept in an oven (Binder, Germany) at $60^{\circ} \mathrm{C}$ for 24 hours. After 24 hours, microspheres were recovered from the vials and preserved in a desiccator until further study.

\section{RESULTS AND DISCUSSION}

Table 1 shows the percentage yield and drug entrapment properties of the optimized microspheres. Yield values ranging from $70-90 \%$ were found suggesting good amount of microspheres were recovered by the adopted technique. Percent DS loading value of $48.42 \%$ for blank suggests that more polymer than drug was present in this batch. Conversely, bathes comprising PEG showed DS loading value of $>50 \%$ suggesting that amount of drug in these batches was more than the amount of polymer. Higher drug loading results in better encapsulation efficiency and this property of microspheres is clearly reflected in the corresponding values of percent encapsulation efficiency (EE) of batch P3-P15. Maximum 99.3\% EE was found for the batch P15 containing maximum amount of PEG. This might be attributed to good solubility of PEG in methanol which might pull more drug particles inward the microsphere matrix and therefore facilitated more drug molecules to be entrapped within the microsphere matrix. ${ }^{22,23}$ In contrast, incorporation of talc in the internal phase resulted in less DS loading and less EE. It might be due to the fact that talc is practically insoluble in methanol which might impede the drug particles to be incorporated within the polymer matrix. ${ }^{23}$ Microspheres obtained were within the size range of 360-831 $\mu \mathrm{m}$ (by population) or 497-922 $\mu \mathrm{m}$ (by volume). Microspheres comprising PEG were larger than those comprising talc. Entrapment of comparatively more drug particles might be attributed to this. A comparatively closer value of diameter-by-population and diameter-by-volume indicates about more uniform size distribution of the microspheres. A comparatively smaller value of 'span' also confirms about this. Considering these 
two parameters, it can be said that batch P15 was the one showing the most uniform size distribution of the microspheres. This is also evident from figure 1 showing size distribution pattern of the optimized batches. From the figure, it is clearly seen that batch P15 showed a unimodal size distribution curve suggesting a good uniformity within the microsphere sizes.

Table 1. Effects of PEG 6000 and talc on microsphere properties (drug: polymer, 1:1; 20\% wt/wt solvent; 1\% wt/wt emulsifier; 3000 rpm).

\begin{tabular}{|c|c|c|c|c|c|c|c|}
\hline \multirow[t]{2}{*}{ Batch } & \multirow{2}{*}{$\begin{array}{l}\text { Amount of } \\
\text { PEG/Talc* }\end{array}$} & \multirow{2}{*}{$\begin{array}{l}\% \\
\text { Yield }\end{array}$} & \multirow{2}{*}{$\begin{array}{l}\text { \% DS } \\
\text { loading }\end{array}$} & \multirow{2}{*}{$\begin{array}{l}\text { \% Encapsulation } \\
\text { efficiency }\end{array}$} & \multicolumn{3}{|c|}{ Mean Particle Size } \\
\hline & & & & & $\begin{array}{l}\text { Population mean } \\
\text { diameter }(\mu \mathrm{m}) \pm S D\end{array}$ & $\begin{array}{l}\text { Volume mean diameter } \\
(\mu \mathrm{m}) \pm \mathrm{SD}\end{array}$ & Span \\
\hline Blank & $0 \%$ & 71.5 & 43.42 & 69.25 & $421.9 \pm 3.1$ & $616.7 \pm 3.8$ & 1.3 \\
\hline P3 & $3 \%$ & 82.8 & 50.7 & 85.7 & $360.3 \pm 8.5$ & $497.1 \pm 3.1$ & 1.1 \\
\hline P5 & $5 \%$ & 75.0 & 57.1 & 96.5 & $508.2 \pm 5.1$ & $657.7 \pm 6.1$ & 0.9 \\
\hline P10 & $10 \%$ & 87.5 & 54.0 & 94.5 & $506.6 \pm 5.7$ & $636.2 \pm 3.1$ & 0.9 \\
\hline P15 & $15 \%$ & 89.3 & 68.1 & 99.3 & $831.7 \pm 2.3$ & $922 . \pm 4.2$ & 0.4 \\
\hline T3 & $3 \%$ & 78.8 & 43.8 & 70.2 & $470.3 \pm 4.5$ & $603 \pm 5.1$ & 1.6 \\
\hline $\mathrm{T} 5$ & $5 \%$ & 80.6 & 43.5 & 68.7 & $475.2 \pm 3.1$ & $744.7 \pm 5.1$ & 1.9 \\
\hline $\mathrm{T} 10$ & $10 \%$ & 83.8 & 44.1 & 71.5 & $515.6 \pm 1.7$ & $745.0 \pm 9.1$ & 1.5 \\
\hline T15 & $15 \%$ & 88.7 & 43.2 & 67.5 & $478.7 \pm 2.7$ & $710.8 \pm 1.2$ & 1.5 \\
\hline
\end{tabular}

* wt/wt of total amount of KSR

Table 2. Correlation coefficient $\left(r^{2}\right)$, reaction constant $(\mathrm{K})$ and diffusion exponent $(\mathrm{n})$ of the model equations applied to the release data of DS.

\begin{tabular}{cccccccccc}
\hline \multirow{2}{*}{ Batch } & \multicolumn{2}{c}{ Zero Order } & \multicolumn{2}{c}{ First Order } & \multicolumn{2}{c}{ Higuchi Model } & \multicolumn{3}{c}{ Peppas-Korsmeyer } \\
\cline { 2 - 10 } & $\mathrm{r}^{2}$ & $\mathrm{~K}_{0}\left(\% \mathrm{~h}^{-1}\right)$ & $\mathrm{r}^{2}$ & $\mathrm{~K}_{1}\left(\% \mathrm{~h}^{-1}\right)$ & $\mathrm{r}^{2}$ & $\mathrm{~K}\left(\% \mathrm{~h}^{0.5}\right)$ & $\mathrm{r}^{2}$ & $\mathrm{~K}_{\mathrm{P}}\left(\% \mathrm{~h}^{-\mathrm{n}}\right)$ & $\mathrm{n}$ \\
\hline Blank & 0.65 & 7.69 & 0.92 & 0.14 & 0.82 & 25.18 & $\mathbf{0 . 9 4}$ & 0.648 & 0.18 \\
P3 & 0.57 & 6.50 & 0.83 & 0.08 & 0.75 & 21.89 & $\mathbf{0 . 9 1}$ & 0.628 & 0.16 \\
P5 & 0.69 & 7.10 & 0.91 & 0.08 & 0.85 & 23.11 & $\mathbf{0 . 9 8}$ & 0.574 & 0.19 \\
P10 & 0.78 & 7.24 & 0.93 & 0.07 & 0.91 & 22.95 & $\mathbf{0 . 9 9}$ & 0.483 & 0.23 \\
P15 & 0.78 & 6.46 & 0.92 & 0.05 & 0.91 & 20.41 & $\mathbf{0 . 9 8}$ & 0.445 & 0.21 \\
T3 & 0.63 & 7.13 & 0.89 & 0.10 & 0.8 & 23.52 & $\mathbf{0 . 9 2}$ & 0.626 & 0.18 \\
T5 & 0.60 & 6.64 & 0.80 & 0.09 & 0.76 & 21.91 & $\mathbf{0 . 8 7}$ & 0.606 & 0.17 \\
T10 & 0.60 & 6.26 & 0.80 & 0.06 & 0.77 & 20.81 & $\mathbf{0 . 8 7}$ & 0.552 & 0.18 \\
T15 & 0.68 & 6.67 & 0.84 & 0.06 & 0.85 & 21.88 & $\mathbf{0 . 9 4}$ & 0.479 & 0.24 \\
Voltalin & $\mathbf{0 . 9 8}$ & 9.69 & $\mathbf{0 . 9 8}$ & 0.07 & $\mathbf{0 . 9 8}$ & 28.55 & $\mathbf{0 . 9 8}$ & 0.184 & 0.71 \\
\hline
\end{tabular}

Best fit in Bold

Figure 2 shows the differential scanning thermograms of the individual compound and the optimized batches. The DSC thermogram of pure DS showed two endothermic peaks (Figure 2a) which were at $56^{\circ} \mathrm{C}$ and $280^{\circ} \mathrm{C}$ and one exothermic peak at $296^{\circ}$ C. KSR showed its characteristic endothermic peak at around $48^{\circ} \mathrm{C}$ (Figure 2b). Talc and PEG also showed their characteristic peak (Figure 2c and 2d). In case of the optimized batches (blank, T15, P15), characteristic peak of DS was present (Figure 2e, 2f, $2 \mathrm{~g}$ ) suggesting no interaction between the drug and the release modifiers. The first small endothermic peak of DS at $56^{\circ} \mathrm{C}$ might be due to loss of water.
The second sharp endothermic peak at around $280^{\circ} \mathrm{C}$ and subsequent exothermic peak around $296^{\circ} \mathrm{C}$ indicate fusion of the solvated crystals and the oxidation reaction between DS and air oxygen, respectively. Endothermic peak of KSR around $48^{\circ} \mathrm{C}$ was found in the thermogram of the respective batches (blank, P15 and T15) also. But peaks of DS and KSR in the optimized batches were less sharp than their original peak as observed in the individual thermograms. Relative blunting of the peaks of DS might be due to the plasticization property of KSR at the ambient temperature. ${ }^{13}$ Particularly, in case of P15, characteristic peak of DS was more blunted than 
others. Subsequent solubilization of DS and entrapment inside the PEG might be the reason. Plasticizer property of PEG might also attribute to this. $^{23}$
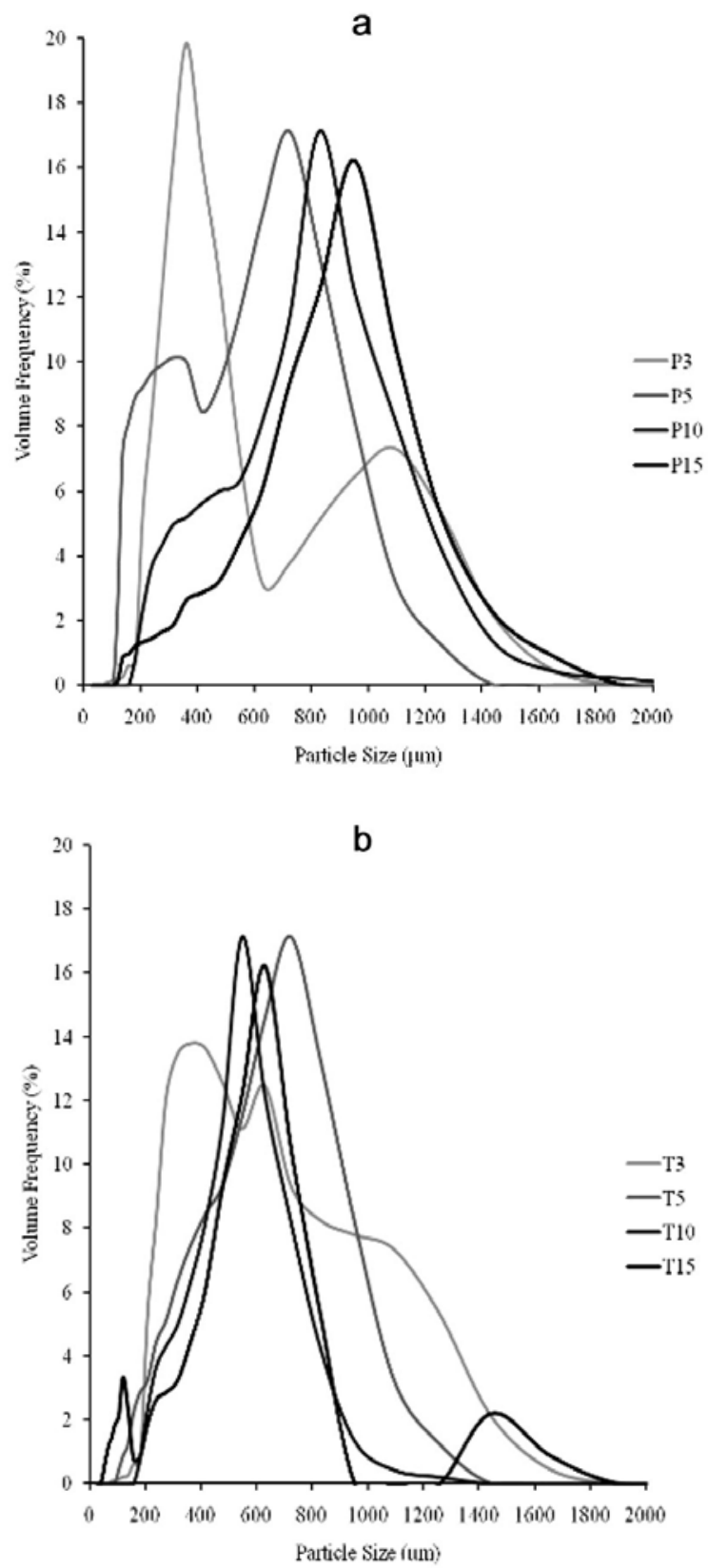

Figure 1. Particle size distribution by volume of four batches of the microspheres prepared with PEG 6000 (a) and talc (b) (core:polymer ratio, 1:1)

The influence of PEG and talc on release profile of DS is shown in figure 3. From the figure, it is clearly seen that increased PEG content resulted in decreased DS release (Figure 3A). After 1 hour of dissolution, approximately 69\% DS was released from microspheres containing no PEG or talc (blank) and after 8 hours, it was approximately 97\%. As PEG was incorporated at an increasing order in the formulations, the percent release of DS was found to

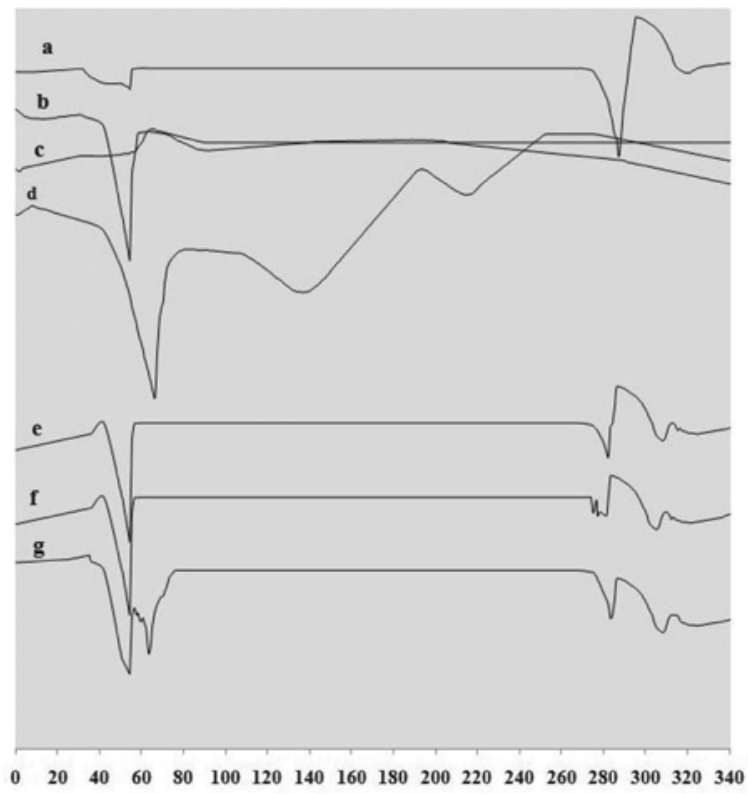

Figure 2. DSC thermograms of DS (a), KSR(b), talc (c), PEG 6000 (d), blank (e), optimized batch T15 (f), and P15 (g).

be lowered significantly from P3 to P15. In case of P3, DS release was approximately $72 \%$ after 1 hour and approximately $89 \%$ after 8 hours of dissolution. In contrast, only 44\% DS was released from P15 after 1 hour and 74\% after 8 hours of dissolution. This decreased drug release due to the incorporation of PEG might be attributed to few reasons. Firstly, incorporation of PEG might render the microspherematrix little elastic in nature as PEG is very good plasticizer and this effect was maximum for P15. Surface appeared as likely to be fused and this is more clear at higher magnification (Figure 4). Secondly, incorporation of PEG resulted in larger microspheres, which have been evident earlier. Due to size enlargement in the microspheres, drug particles had to travel comparatively long distance inside those larger particles which eventually slowed down the release of DS..$^{24,25}$ 

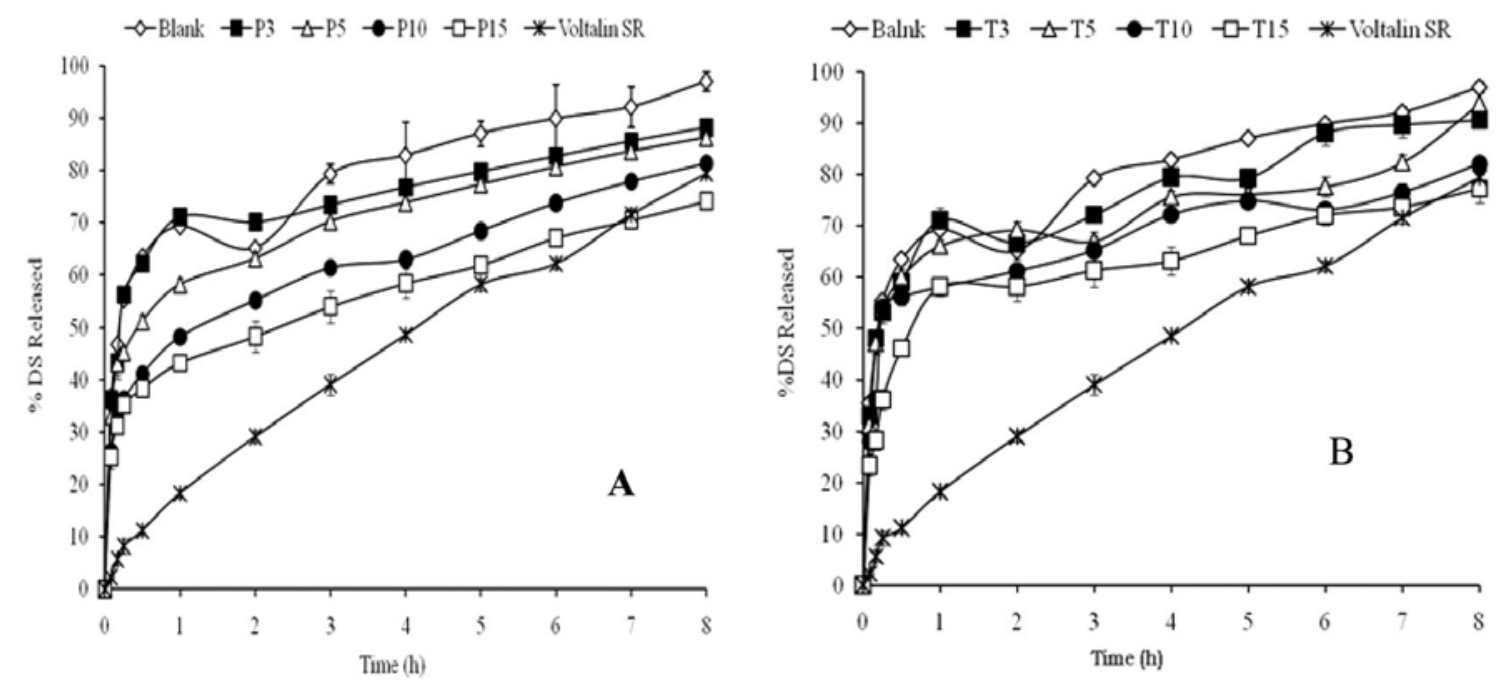

Figure 3. Release profile of DS from KSR microspheres prepared using difwhere talc was used at different concentrations: $3 \%$ (T3), $5 \%$ (T5), $10 \%$ (T10) and 15\% (T15), $\mathrm{n}=6$.
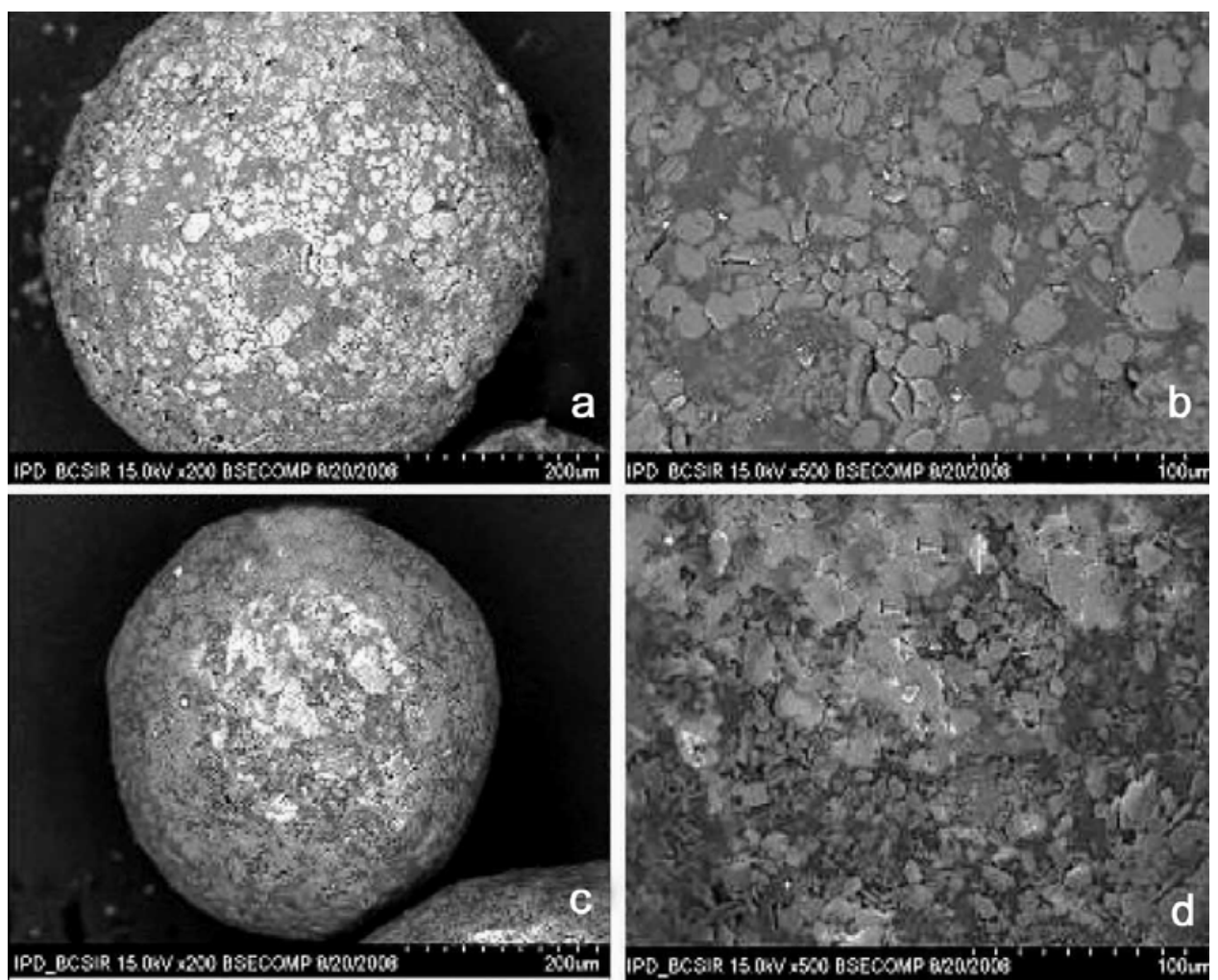

Figure 4. Scanning electron microscopic photograph of the microspheres 15\% PEG (a,b) and talc (c,d).

Effect of talc on release profile of DS is shown in figure 3B. Though release of DS was found to be slowed down gradually from $\mathrm{T} 3$ to $\mathrm{T} 15$, it was comparatively at smaller extents than those by PEG. DS release was 58\% after 1 hour and 77\% after 8 hours of dissolution in case T15. Unlike PEG, initial burst release was less controlled by the presence of talc. But an overall reduction was achieved. Molecular structure and solubility of talc might be accounted for this controlled release of the drug. ${ }^{22}$ 
That is, crystalline flat-shaped talc molecules might render the microsphere particle a compact matrix and this compact nature of the microsphere is clearly viewed in figure 4 . So, when the microspheres were immersed in the dissolution media, surface DS particles immediately left the matrix resulting the immediate burst release, even though further migration of DS crystals might be impeded due to the presence of talc crystals. In addition, being hydrophobic in nature, talc might also repel the travel of dissolution media toward the microsphere matrix. These two events might collectively hinder the release of DS and resulted a comparatively slower release of DS. Fassihi et al (1994), Fassihi et al (1996) and Schultz et al (1997) also reported about such release retarding property of talc. ${ }^{26-28}$

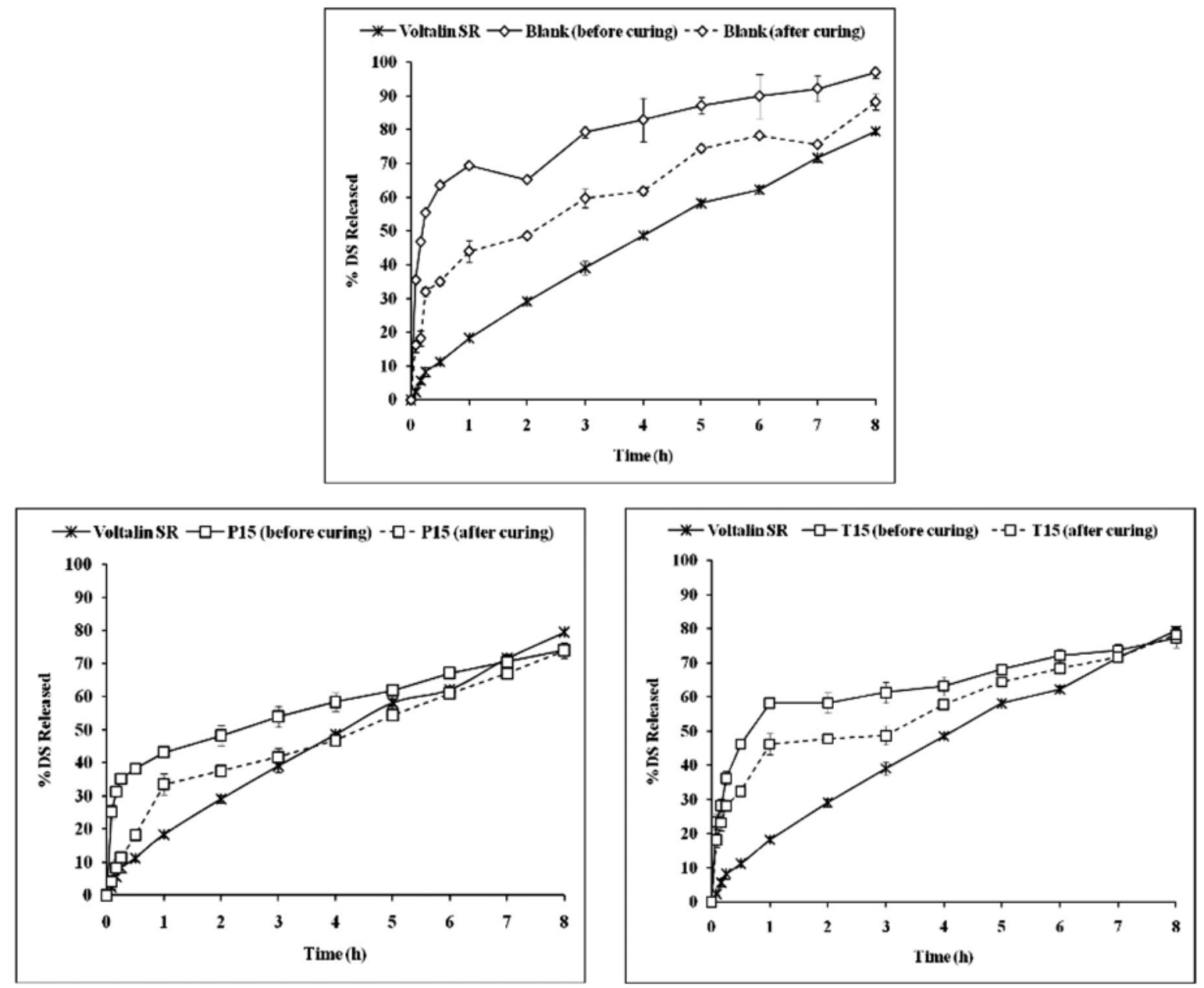

Figure 5. Comparison of DS release profile from formulated microspheres before and after curing $(n=6)$.

Effect of heating or annealing on release profile of DS is shown in figure 5. Remarkable reduction in the DS release was observed after annealing. When amorphous polymers are heated to temperature above glass transition temperature (Tg), its mechanical properties are influenced. This process is called annealing or curing. Annealing increases the density within the polymer and decreases the rate of stress relaxation at temperature below the $\mathrm{Tg}$, This change tend to improve the dimensional stability of the polymer. Annealing can significantly affect drug release from particles prepared using polymers of low $\mathrm{T}_{\mathrm{g}} \cdot{ }^{17-19}$ Omelczuk et al also mentioned that curing above the $\mathrm{T} g$ of a polymer significantly improves 
film formation, which therefore, reduces the permeability of the film and avoids accelerated and irreproducible dissolution result. ${ }^{19}$ KSR, which contains $80 \%$ of polyvinyl acetate (PVA) polymer, is amorphous in nature and has a low $\mathrm{T}_{\mathrm{g}}$ of $28-31^{\circ} \mathrm{C}$. When used in the tablet formation, it undergoes little cured during the normal processing conditions and, therefore, affects physical integrity and drug release rate. $^{11,14}$ Shao et al. also reported that curing after compression is very critical in stabilizing the release

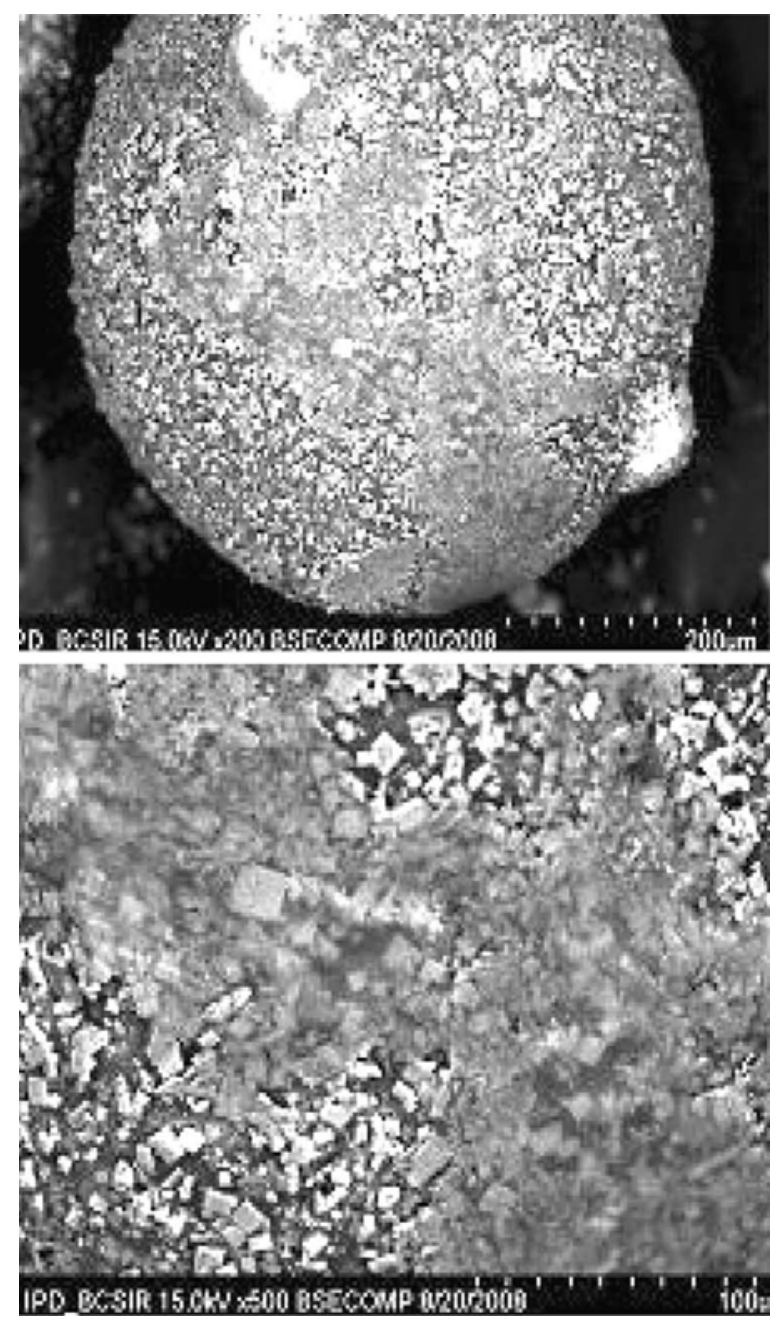

Figure 6. SEM photograph of batch P15 after curing.

rates of dosage form containing high levels (more than $47 \%$ ) of Kollidon ${ }^{\circledR}$ SR. A few hours of heating at $60^{\circ} \mathrm{C}$ is a sufficient curing-condition for such tablets. ${ }^{14}$ Thus, in order to get a stabilized dissolution pattern of DS and improved physical stability, microspheres were subjected to curing at $60^{\circ} \mathrm{C}$ for 24 hours. Cumulative percent releases of DS were found to be decreased (Figure 5) after curing. Importantly, immediate burst release was found to be lowered after curing. Microspheres containing no PEG (blank) showed $70 \%$ release at the end of 1 hour before curing and 44\% release after curing. Batch P15 showed $43 \%$ release of DS before curing and 33\% release after curing. Similarly, T15 showed 59\% release before curing and $47 \%$ release after curing. This reduced drug release after curing might be due to the adequate changes in the surface of the cured microspheres, which can be seen in figure 6. Surface appeared more fused in nature. In fact, surface particles were likely to be embedded within the fused portion of the surface.

Release data were fitted in different kinetic equations to determine the mechanism of DS release. Kinetic parameters are shown in table 2. A value of $n$ $<0.45$ indicates fickian (case I) release; > 0.45 but $<$ 0.89 indicates non-fickian (anomalous) release; and $>$ 0.89 indicates super case II type of release. Case I refers to diffusion controlled drug release, case II refers to the erosion of the polymeric chain, and anomalous transport (non-fickian) refers to a combination of both diffusion as well as erosion controlled drug release. ${ }^{29}$ So, it is clearly seen that case I type of release mechanism was predominant in case of DS release from the formulated microspheres and hence, the underlying mechanism of DS release was diffusion-controlled drug release.

\section{CONCLUSION}

It can be inferred from the above findings that Kollidon ${ }^{\circledR}$ SR can be a good choice of polymer to prepare sustained release multiparticulate system of water soluble drugs like diclofenac sodium. Incorporation of PEG and talc also showed good release controlling property and therefore, these two excipients can be used to prepare sustained release microparticulate systems. Annealing also showed good effectiveness in reducing the release of the drug. So, it can be concluded that the technique adopted is very feasible and effective to prepare sustained release microspheres of water soluble drugs 
and release modifiers like PEG 6000 or talc or postmicroencapsulation technique like annealing may further facilitate the process of achieving sustained release action.

\section{ACKNOWLEDGEMENTS}

The authors would like to thank Renata Ltd., Bangladesh, Incepta Pharmaceuticals Ltd., Bangladesh and Bangladesh Council of Scientific and Industrial Research (BCSIR), Bangladesh for providing with instrumental facilities to carry out this research work. They also thank Square Pharmaceuticals, Bangladesh for their generous support with chemicals.

\section{REFERENCES}

1. Cooke, P.M. 1988. Side-effects of non-steroidal antiinflammatory drugs. Med. J. Aust. 148, 248-251.

2. Deasy, P.B. 1984. Microencapsulation and Related Drug Processes (New York: Marcel Dekker, Inc.), p. 361.

3. Gohel, M.C. and Amin, A.F. 1999. Formulation design and optimization of modified-release microspheres of diclofenac sodium. Drug. Dev. Ind. Pharm. 25, 247-251.

4. Perumal, D. 2001. Microencapsulation of ibuprofen and Eudragi RS 100 by the emulsion solvent diffusion technique. Int. J. Pharm. 218, 1-11.

5. Biju, S.S., Saisivam, S. and Rajan, N.S. 2004. Dual coated erodible microparticles for modified release of diclofenac sodium. Eur. J. Pharm. Biopharm. 58, 61-67.

6. Gilman, A.G., Ralf, T.W. and Nile, A.S. 2001. The pharmacological basis of therapeutics: Volume I, 10th ed. Maxwell Publishing Corporation. p. 709.

7. Jani, G.K. and Gohel, M.C. 1997. Effects of selected formulation parameters on the entrapment of diclofenac sodium in ethyl cellulose microspheres. J. Control. Release. 43, 245-250.

8. Amin, A.F. and Gohel, M.C. 1998. Formulation optimization of controlled release diclofenac sodium microspheres using factorial design. J. Control. Release. 51, 115-122.

9. Watts, P.J., Davies, M.C. and Melia, C.D. 1990. Microencapsulation using emulsification solvent evaporation: an overview of techniques and applications. Crit. Rev. Ther. Drug. Carrier Syst. 7, 235-259.

10. Jalil, R. and Nixon, J.R. 1990. Biodegradable poly (lactic acid) and poly (lactide-co-glycolide) microcapsules: problems associated with preparative techniques and release properties. J. Microencapsul. 7: 297-325.
11. BASF, Technical Information, April 2006.

12. Hauschild, K. and Picker-Freyer, K.M. 2006. Evaluation of tableting and tablet properties of Kollidon SR: the influence of moisture and mixtures with theophylline monohydrate. Pharm. Dev. Tech. 11, 125-40.

13. Kranz, H., Le Brun, V. and Wagner, T. 2005. Development of a multi-particulate extended release formulation for ZK 811 752, a weakly basic drug. Int. J. Pharm. 299, 84-91.

14. Shao, Z.J., Farooqi, M.I., Diaz, S., Krishna, A.K. and Muhammad, N.A. 2001. Effect of formulation variables and post-compression curing on drug release from a new sustained-release matrix material: polyvinylacetate-povidone. Pharm. Dev. Tech. 6, 247-254.

15. Islam, M.S., Alam, S.M.R., Sadat, S.M.A., Chowdhury, .JA. and Jalil, R. 2009. Preparation and characterization of Polyvinyl Acetate (Kollidon ${ }^{\circledR}$ SR) microspheres containing diclofenac sodium I: effect of stirring rate and total solid content. Dhaka Univ. J. Pharma. Sci. 8, 111-116.

16. Islam, M.S., Rahman, A., Islam, M.K., Chowdhury, J.A. and Jalil, R. 2009. Preparation and characterization of polyvinyl acetate (Kollidon ${ }^{\circledR}$ SR) microspheres containing diclofenac sodium II: effect of core loading. Dhaka Univ. J. Pharma. Sci. 8, 117-122.

17. Kader, A. and Jalil, R. 1998a. Effect of physicochemical factors on the release kinetics of hydrophilic drugs from poly(L-lactic acid) (L-PLA) pellets. Drug Dev. Ind. Pharm. 24, 535-9.

18. Kader, A. and Jalil, R. 1998b. In vitro release of theophylline from poly(lactic acid) sustained-release pellets prepared by direct compression. Drug Dev. Ind. Pharm. 24, 527-34.

19. Omelczuk, M.O. and Mcginity, J.W. 1993. The influence of thermal treatment on the physical-mechanical and dissolution properties of tablets containing poly(DL-lactic acid). Pharm. Res. 10, 542-8.

20. Florey, K. 1990. Analytical profiles of drug substances, Academic Press Inc., New York. 19, 123.

21. Attama, A.A. and Mpamaugo, V.E. 2006. Pharmacodynamics of piroxicam from self-emulsifying lipospheres formulated with homolipids extracted from Capra hircus, Drug Deliv. 13, 133-137.

22. Kibbe, A.H. 2000. Handbook of Pharmaceutical Excipients. $3^{\text {rd }}$ Ed. pp. 511-514, 555-557.

23. Wells, J.I., Bhatt, D.A. and Khan, K.A. 1982. Improved wet massed tabletting using plasticized binders. J. Pharm. Pharmacol. 34, 46.

24. Kimi, B.K., Hwang, S.J., Parks, J.B. and Park, H.J. 2002. Preparation and characterization of drug-loaded 
Polymethacrylate microspheres by an emulsion solvent evaporation method. J. Microencapsul. 19, 811-822.

25. Lee, J.H,, Park, T.G. and Choi, H.K. 2000. Effect of formulation and processing variables on the characteristics of microspheres for water soluble drugs prepared by w/o/o double emulsion solvent diffusion method. Int. J. Pharm. 196, 75-83.

26. Fassihi, R.A., Mcphillips, A.M., Uraizee, S.A. and Sakr, A.M. 1994. Potential use of magnesium stearate and talc as dissolution retardant in the development of controlled release drug delivery systems. Pharm. Ind. 56, 579-583.

27. Fassihi, R., Fabian, J. and Sakr, A.M. 1996. Applicaion of response surface methodology to design optimization in formulation of a typical controlled release systems. Drugs Made Ger. 39, 122-126.

28. Schultz, P., Tho, I. and Kleinebudde, P. 1997. New multiparticulate delayed release system. Part 2. Coating formulation and properties of free films. J. Control Release. 47, 191-199.

29. Peppas, N.A. 1985. Analysis of Fickian and non-Fickian drug release from polymers. Pharm. Acta Helv. 60, 110-111. 